\title{
EFEITOS DA INTENSIDADE, NO TREINAMENTO RESISTIDO, SOBRE APERCEPÇÃO DE AFETO EM IDOSOS: UMA REVISÃO SISTEMÁTICA
}

\author{
${ }^{1}$ David Barbosa da Silva, ${ }^{1} J o s e ́$ Samuel de Vasconcelos Sobral, ${ }^{1}$ Tays Kettyman Alves Galdino da \\ Silva, ${ }^{2}$ Luciano Machado Ferreira Tenório de Oliveira, ${ }^{3}$ André Luiz Torres Pirauá.
}

\section{RESUMO}

Introdução: O envelhecimento humano é um processo natural e gradual. Uma das formas de minimizar esse processo se dá através do exercício físico, alcançando melhorias físicas, cognitivas e psicológicas. O Treinamento Resistido (TR) tem sido recomendado para população idosa, pois ele é capaz de produzir respostas físicas positivas além da qualidade de vida, humor e melhora da ansiedade. Objetivo: Este artigo teve como objetivo investigar os efeitos da intensidade, no treinamento resistido (TR) sobre a percepção de afeto em idosos. Método: Trata-se de uma revisão sistemática em que as bases de dados utilizadas para a pesquisa foram: Pubmed, Scorpus e Science Direct. A busca e seleção dos artigos ocorreram mediante descritores consultados na base de dados Medical Subject Headings (MeSH). O período de busca e seleção dos artigos compreendeu os meses de abril e maio de 2018, conduzido por pesquisadores de forma independente, em três estágios: análise dos títulos, leitura dos resumos e análise dos artigos completos. Resultados e Discussão: Foram identificados 8 estudos que obtiveram respostas afetivas nas variações de intensidade do TR. Observou-se que o resultado afetivo foi positivo levando em consideração a intensidade de moderada a alta, na grande maioria dos artigos. Conclusão: $O$ presente estudo demonstrou que a intensidade deve ser prescrita levando em consideração não apenas as respostas fisiológicas como também as afetivas, para que haja uma maior aderência ao TR em idosos.

Palavras-Chave: Idoso, Treinamento Resistido. Intensidade, Percepção de Afeto.

\section{EFFECTS OF INTENSITY IN RESISTANCE TRAINING ON AFFECT APPROACH IN ELDERLY: A SYSTEMATIC REVIEW}

\section{ABSTRACT}

Introduction: The human process is a natural and gradual process. The insulines from the physical physical treatment from physical physics, reaching physical, cognitive and psychological. Resistance Training (TR) has been recommended for the elderly population, because it is able to promote the action of promoting desires, in addition to quality of life, mood and anxiety improvement. Objective: This article aimed to investigate the effects of intensity without resistance training (TR) on the perception of a goal in the elderly. Method: This was a systematic review in databases used for research were: Pubmed, Scorpus and Science Direct. The Medical Subject Headings (MeSH). The year of search and publication of articles was held in May 2018, conducted by researchers independently, in three stages: analysis of titles, reading of abstracts and analysis of complete articles. Results and Discussion: We identified 8 studies that obtained the understanding of the TR intensity variables. Response time was positive, leading to moderate to high intensity in most articles. Conclusion: The present study demonstrated that error should be prescribed as a measure of physical and mental endurance.

Key words: Elderly, Resistance training, Intensity, Perception of affect.

1 - Centro Universitário Tabosa de Almeida- ASCES- UNITA 2 - Universidade Federal de Pernambuco- Centro Acadêmirn do Vitória 2 - I Inivorcidano Fodoral Rural de Pernambuco- UFRPE. ASCES-UNITA: Av. Portuge Treinamento resistido e idosos 16-400, telefone: (81) 2103-2000.

E-mail: davidupe@live.com. 
INTRODUÇÃO

O envelhecimento humano é um processo natural e gradual que envolve degradação dos sistemas fisiológicos (SCHNEIDER; IRIGARAY, 2008). Tal processo não está ligado apenas a condição genética, mas, sobretudo aos hábitos do individuo ao longo da vida (FECHINE; TROMPIERE, 2012). Além disso, repercute sobre as dimensões psíquicas, cognitivas, personalidade e afeto (FECHINI; TROMPIERE, 2012).

Uma das estratégias para atenuar os efeitos deletérios do envelhecimento se dá por meio de exercícios, tornando-se cada vez mais importante para idosos, pois promove melhora das funções psicológicas e fisiológicas (BLUMENTHAL et.al., 1989). Embora os benefícios da prática regular de exercícios físicos sejam amplamente reconhecidos, apenas $8,5 \%$ dos idosos atendem as recomendações, acumulando 150 minutos semanais com intensidade moderada e/ou vigorosa (MATSUDO; MATSUDO; BARROS NETO, 2001). Além das respostas fisiológicas e funcionais, a prática de exercício promove modulações nas respostas afetivas, ou seja, em manifestações de emoções envolvendo sensações de prazer ou desprazer (EKKEKAKIS; HALL; PETRUZZELLO, 2005).

A percepção de prazer/desprazer está significativamente associada com a percepção de esforço (HARDY; REJESK, 1989). De modo geral, exercícios praticados em intensidades vigorosas, promovem grande desconforto (EKKEKAKIS; LIND, 2006). Nesse sentido, exercícios percebidos como intensos produzem respostas afetivas negativas, contribuindo para o abandono do praticante, sendo essas respostas evidenciadas em exercícios aeróbios (EKKEKAKIS; HALL; PETRUZZELLO, 2005).

Paralelamente, observa-se que 0 treinamento resistido TR, tem sido recomendado para o idoso, por ser um método capaz de aumentar força e potencia muscular, além de aumentar a densidade mineral óssea (ANTONINI; LIBERALI; DA CRUZ, 2010), prevenir e/ou atenuar aspectos deletérios como sarcopenia e dinapenia, entre outros agravos (ZACARON et al., 2006). Adicionalmente, Cassilhas et al., (2010) relata que o TR proporciona melhoria da qualidade de vida, humor, ansiedade, qualidade do sono e massa muscular.

Ciente de que as recomendações específicas sobre a prática do TR para o idoso, preconizadas pelo ACSM (American College Of Sports Medicine, 2009), incluem a realização de intensidades moderadas (5-6) a vigorosas (7-8) em uma escala de 0 a 10, correspondente a 50 a $80 \%$ de uma repetição máxima, é possível especular que essas recomendações, relativas a intensidade, impliquem em percepções negativas de afeto do idoso durante o TR e, consequentemente, sobre sua aderência ao treinamento. Diante do exposto, e da ausência de estudos de revisão acerca dos efeitos da intensidade sobre a percepção de afeto em idosos, durante a realização do TR, o objetivo do presente estudo é avaliar, através de uma revisão sistemática, os efeitos da intensidade, no TR, sobre as respostas afetivas em idosos. 


\section{MÉTODO}

Trata-se de uma revisão sistemática de estudos originais que analisaram a relação entre percepção afetiva com a prática de TR em idosos. Para a presente revisão foram incluídos os artigos indexados nas bases de dados Pubmed, Scopus e Science Direct. Para a consulta nas referidas bases de dados, foram considerados descritores consultados na base de dados Medical Subject Headings (MeSH). O período de busca e seleção dos artigos compreendeu os meses de abril e maio de 2018.

Como critérios de inclusão foram utilizados: ensaios clínicos randomizados, pessoas a partir dos 60 anos, ambos os sexos e publicados na língua inglesa. Artigos duplicados, presentes em mais de uma base de dados, foram excluídos do estudo. Todo o processo de seleção e busca dos artigos foi conduzido por pesquisadores de forma independente, em três estágios: 1) análise dos títulos, 2) resumos e 3) artigos completos. Em cada uma das etapas os resultados foram confrontados entre os avaliadores, a fim de verificar a concordância entre os pares. Os descritores foram divididos em 3 blocos, apresentados na figura 1.

\section{RESULTADOS}

$\mathrm{Na}$ primeira fase foram selecionados 9141 artigos, na segunda 632, foram relacionados 24 artigos, onde passou para 8 na etapa de discussão dos resultados, de forma a contemplar os estudos com a temática proposta, de acordo com a figura 2.

Figura 1 - Expressão de busca criada a partir dos descritores consultados na base de dados MeSH.

\begin{tabular}{|c|}
\hline IDOSO \\
\hline MeSH \\
\hline Elderly OR \\
Erderly OR \\
Frail OR \\
Frail Elders OR \\
ElderOR \\
Frail Elders OR \\
Frail OR \\
Frail Elder OR \\
Functionally-Impaired OR \\
Functionally-Impaired OR \\
Functionally Impaired \\
Elderly OR \\
Frail older Adults OR \\
Adult Frail Older OR \\
Addult OR \\
Frail Older OR \\
Adult Older OR \\
Adult Frail Older OR \\
Adults,Frail \\
\hline
\end{tabular}

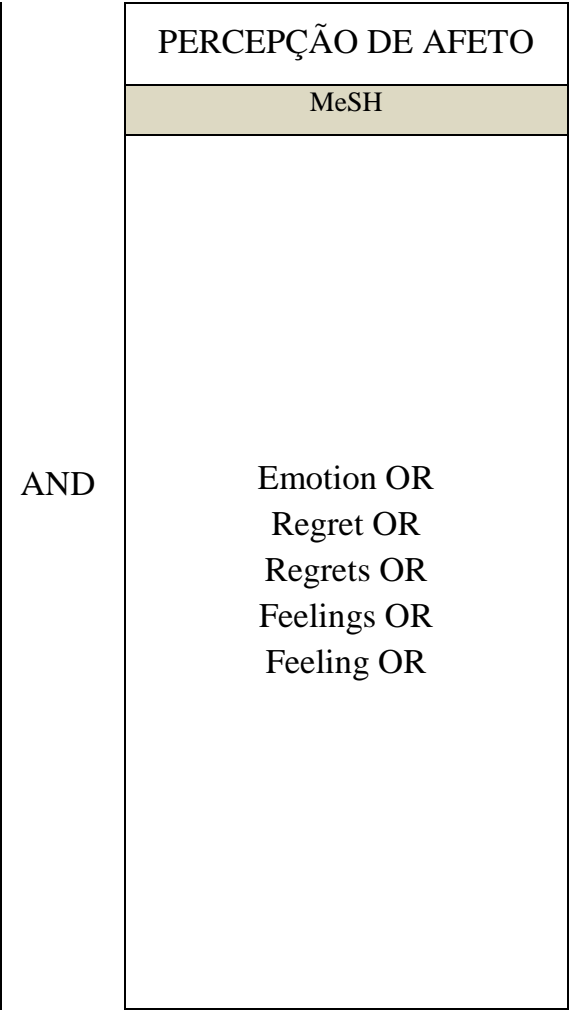

EXERCÍCIO RESISTIDO

\begin{tabular}{|c|}
\hline $\mathrm{MeSH}$ \\
\hline
\end{tabular}

Trainings OR, Exercise

Training OR, Exercise OR

Exercise Trainings OR

Exercise Training OR

AND Exercises OR Exercise

Isometric OR Exercises OR

Exercises Isometric OR

Exercise Isometric OR

Exercises Acute OR

Exercise Acute OR Acute

Exercises OR Acute

Exercise OR Physical

Exercises OR Physical

Exercise OR Exercises, Physical 

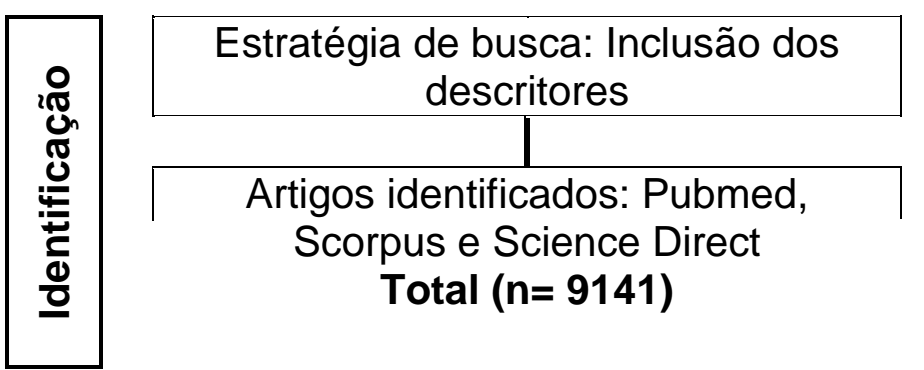

Scorpus e Science Direct

Total $(n=9141)$
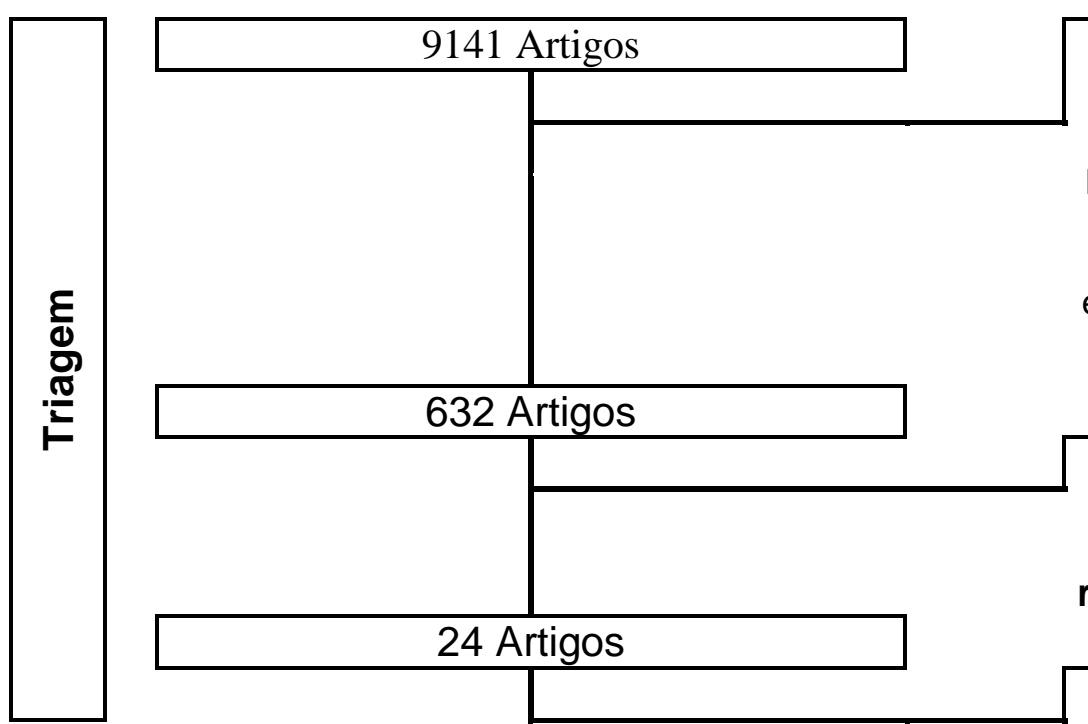

Estudos

excluídos após

leitura dos títulos

$(n=7295)$

Artigos repetidos

excluídos $(n=1214)$

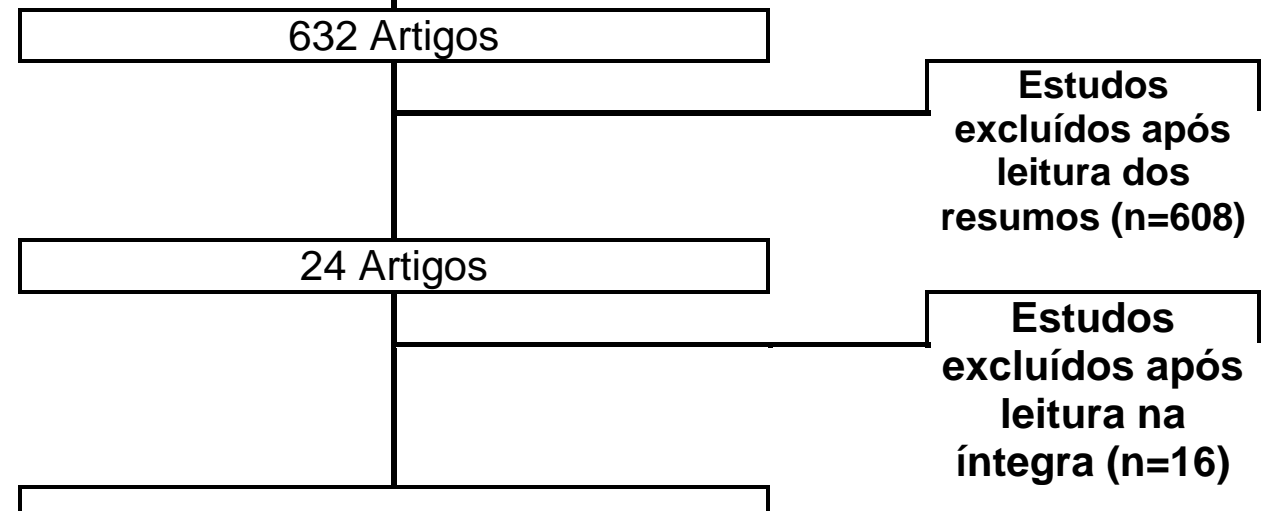

\section{8 artigos incluídos}

Figura 2- Organograma de busca e seleção de artigos. 


\begin{tabular}{|c|c|c|c|c|c|}
\hline $\begin{array}{l}\text { AUTOR/ ANO DE } \\
\text { PUBLICAÇÃO }\end{array}$ & $\begin{array}{c}\text { TIPO DE } \\
\text { ESTUDO E } \\
\text { DURAÇÃO }\end{array}$ & $\begin{array}{c}\text { CARACTERÍS } \\
\text { TICAS DAS } \\
\text { AMOSTRAS } \\
\text { DOS } \\
\text { ESTUDOS }\end{array}$ & $\begin{array}{c}\text { FERRAMENTA } \\
\text { PARA } \\
\text { MENSURAÇÃO } \\
\text { AFETIVA }\end{array}$ & $\begin{array}{l}\text { INTENSIDA } \\
\text { DE } \\
\text { APLICADA } \\
\text { AO TR }\end{array}$ & $\begin{array}{c}\text { RESULTADO } \\
\text { PERCEPÇÃO DE } \\
\text { ESFORÇO AO TR }\end{array}$ \\
\hline $\begin{array}{l}\frac{\text { Lacharité-Lemieux }}{\mathrm{M}, \text { Brunelle }} \\
\underline{\text { Jp}}, \underline{\text { Dionne lj. (2015) }}\end{array}$ & $\begin{array}{c}\text { Ensaio } \\
\text { Aleatório/ } 12 \\
\text { semanas }\end{array}$ & $\begin{array}{l}23 \text { mulheres } \\
\text { saudáveis } \\
\text { pós-menopausa } \\
\text { com idade } \\
\text { média de } 61 \\
\text { anos }\end{array}$ & $\begin{array}{l}\text { Feeling Scale } \\
\text { (FS) }\end{array}$ & $\begin{array}{c}65 \text { a } 95 \% \text { da FC } \\
\text { na duração } \\
\text { máxima de } \\
\text { formação }\end{array}$ & $\begin{array}{l}\text { Percepção de afeto } \\
\text { Positivo }\end{array}$ \\
\hline $\begin{array}{l}\text { Toshihiko, Tsutsumi; } \\
\text { Brian M. Don; } \\
\text { Koji, } \\
\text { Takenaka, } \\
\text { Koichiro } \\
\text { Oka.(1997) }\end{array}$ & $\begin{array}{l}\text { Ensaio clínico } \\
\text { Randomizado } \\
\text { / } 12 \text { semanas }\end{array}$ & $\begin{array}{c}45 \text { idosos (36 } \\
\text { mulheres e } 9 \\
\text { homens) } \\
\text { sedentários com } \\
\text { idade média de } \\
74 \text { anos }\end{array}$ & $\begin{array}{l}\text { Feeling Scale } \\
\text { (FS) }\end{array}$ & $\begin{array}{c}50 \text { a } 90 \% \text { da FC } \\
\text { na duração } \\
\text { máxima }\end{array}$ & $\begin{array}{c}\text { Percepção de afeto } \\
\text { Positivo }\end{array}$ \\
\hline $\begin{array}{c}\text { Toshihiko, } \\
\text { Tsutsumi; } \\
\text { Brian M. Don;Koji, } \\
\text { Leonard D; et al. } \\
\text { (1998) }\end{array}$ & $\begin{array}{l}\text { Ensaio } \\
\text { Aleatório/ } 12 \\
\text { semanas }\end{array}$ & $\begin{array}{l}36 \text { idosos } \\
\text { sedentários com } \\
\text { idade média de } \\
68 \text { anos }\end{array}$ & $\begin{array}{l}\text { Feeling Scale } \\
\text { (FS) }\end{array}$ & $\begin{array}{c}70 \text { a } 95 \% \text { da FC } \\
\text { máx }\end{array}$ & $\begin{array}{c}\text { Percepção de afeto } \\
\text { Positivo }\end{array}$ \\
\hline $\begin{array}{l}\text { Edwards; et al. } \\
\qquad(2008)\end{array}$ & $\begin{array}{l}\text { Piloto/12 } \\
\text { semanas }\end{array}$ & $\begin{array}{c}36 \text { pessoas com } \\
\text { demência } \\
\text { moderada a } \\
\text { grave }\end{array}$ & $\begin{array}{l}\text { Affect Rating } \\
\text { Scale }\end{array}$ & $\begin{array}{c}50 \text { a } 85 \% \text { da FC } \\
\text { máx }\end{array}$ & $\begin{array}{l}\text { Percepção de afeto } \\
\text { Positivo }\end{array}$ \\
\hline $\begin{array}{l}\text { Cassilhas et al. } \\
\qquad(2010)\end{array}$ & $\begin{array}{c}\text { Ensaio } \\
\text { Aleatório/ } 24 \\
\text { semanas }\end{array}$ & $\begin{array}{l}43 \text { homens } \\
\text { sedentários com } \\
\text { idade média de } \\
69 \text { anos }\end{array}$ & $\begin{array}{c}\text { Escala visual } \\
\text { analógica (VAS) }\end{array}$ & $\begin{array}{c}70 \text { a } 98 \% \text { da FC } \\
\text { máx }\end{array}$ & $\begin{array}{c}\text { Percepção de afeto } \\
\text { Positivo }\end{array}$ \\
\hline $\begin{array}{l}\text { Sean P. Mullen et } \\
\text { al. (2011) }\end{array}$ & $\begin{array}{l}\text { Randomizado } \\
\text { Controlado/ } \\
12 \text { meses }\end{array}$ & $\begin{array}{c}179 \text { idosos } \\
\text { sedentários (117 } \\
\text { feminino e } 62 \\
\text { masculino) }\end{array}$ & $\begin{array}{c}\text { Self- } \\
\text { Esteem Scale }\end{array}$ & $\begin{array}{c}50 \text { a } 85 \% \text { da FC } \\
\text { máx }\end{array}$ & $\begin{array}{l}\text { Percepção de afeto } \\
\text { Positivo }\end{array}$ \\
\hline Jette et al. (1996) & $\begin{array}{l}\text { Randomizado } \\
\text { Controlado/ } \\
15 \text { semanas }\end{array}$ & $\begin{array}{l}102 \text { idosos sem } \\
\text { deficiência com } \\
\text { idade média de } \\
71 \text { anos }\end{array}$ & $\begin{array}{l}\text { Feeling Scale } \\
\text { (FS) }\end{array}$ & $\begin{array}{c}40 \text { a } 55 \% \text { da FC } \\
\text { na duração } \\
\text { máxima }\end{array}$ & $\begin{array}{c}\text { Percepção de afeto } \\
\text { Neutro }\end{array}$ \\
\hline $\begin{array}{l}\text { Sd O'shea, Nf Taylor } \\
\text { E Jd Paratz. (2015) }\end{array}$ & $\begin{array}{l}\text { Randomizado } \\
\text { Controlado/ } \\
24 \text { semanas }\end{array}$ & $\begin{array}{l}22 \text { participantes } \\
\text { (14 feminino e } 8 \\
\text { masculino) }\end{array}$ & $\begin{array}{l}\text { Feeling Scale } \\
\text { (FS) }\end{array}$ & $\begin{array}{c}65 \text { a } 85 \% \text { da FC } \\
\text { máx }\end{array}$ & $\begin{array}{l}\text { Percepção de afeto } \\
\text { Positivo }\end{array}$ \\
\hline
\end{tabular}

Quadro 1- Triagem dos artigos selecionados.

O quadro 1 apresentado acima, mostra dados relevantes com a intensidade do
TR e a percepção de afeto. Observou-se que o resultado afetivo foi positivo levando em consideração a intensidade de moderada a 
alta, na maioria dos artigos. Outro achado relevante se deu nos idosos terem sido expostos a uma intensidade moderada a vigorosa, onde perceberam o esforço em menor escala, consequentemente produzindo respostas afetivas positivas.

A ferramenta que foi mais utilizada nos artigos foi a feeling scale de Hardy e Rejeski

\section{DISCUSSÃO}

O objetivo do presente estudo foi avaliar, através de uma revisão sistemática, os efeitos da intensidade, no TR, sobre as respostas afetivas em idosos. Contrapondo o estudo de Ekkekakis, Hall e Petruzzello (2005) que relataram que exercícios praticados intensamente produzem respostas afetivas negativas, contribuindo para o abandono do praticante. Esta presente revisão sistemática evidenciou que as pesquisas que utilizaram intensidades mais elevadas no TR tiveram uma percepção de afeto positiva entre os idosos, assegurando assim a hipótese inicial. Esta intensidade deve obedecer a métodos de percepção de afeto como o feeling scale de Hardy e Rejeski (1989), para sua progressão. Confirmando que a intensidade do TR interfere positivamente nas respostas afetivas nos idosos, em diferentes condições de saúde, físicas e mentais.

Toshihiko et al., (1998) relata que encontrou respostas afetivas positivas em idosos no TR de moderada e alta intensidade, mas ressalta a importância de um estudo mais longo para ratificar tal achado. Seguindo esta linha, Lemieux; Brunelle e Dionne (2015) também evidenciam ganhos de afeto positivo em moderada e alta intensidade e ressalta os benefícios sobre afetividade no TR feitos em ambientes ao ar livre. Paralelamente, Edwards et al., (2008) evidenciou respostas afetivas positivas ao TR em intensidade moderada mesmo em idosos com problemas psicológicos. Tsutsumi; Zaichkowsky e Delizonna (1997) também confirmam a relação positiva afetiva ao TR, mas aponta à baixa e alta intensidade em seu estudo. Ademais, Cassilhas et al., (2010) enfatiza a relação da alta intensidade com o humor, ansiedade, qualidade de vida e sono, não encontrando tal relação com a baixa intensidade, mesmo com o aditivo do soro IGF-1, proteína principalmente hepática, que estimula vários benefícios no corpo.

Em contraste com as intensidades evidenciadas nos estudos anteriores Jette et al., (1996) foi o único que obteve resposta na percepção de afeto neutra. Ressalta-se que
(1989), identificando de forma ágil as respostas afetivas. Escala esta, que têm relação entre +5 "muito bom", 0 "neutro" e -5 "muito ruim" e intermediários: +3 = bom; +1 = razoavelmente bom; -1 = razoavelmente ruim; $-3$

ruim.

respostas afetivas em qualquer intensidade do TR pode ocasionar melhoras físicas, mentais e sociais, além de gerar maior confiança e controle motor, repercutindo positivamente nas atividades diárias (O'SHE; TAYLOR E PARATZ, 2015). Proporcional ao achado de Mullen (2011) que com intensidade de moderada a alta do TR foram obtidas respostas afetivas positivas. Desta forma evidencia a importância do TR em intensidade moderadas e altas nas respostas afetivas positivas em idosos, respeitando a individualidade biológica de cada um.

Interessantemente, Ekkekakis (2003) em sua teoria do modo duplo Ekkekakis, Parfitt e Petruzzello (2011), prediz que na maioria das pessoas as repostas afetivas ao exercício são predominantemente positivas, até o ponto do limiar ventilatório $e$ até $o$ ponto de compensação respiratória. Fatores que são responsáveis pela percepção afetiva em diversas fases do exercício, interagem nas respostas afetivas, onde alguns indivíduos apresentam respostas positivas, e outras negativas. Esta revisão sistemática identificou que o TR tido na intensidade moderada a alta, condiciona melhor os idosos, desse modo tornando-os mais resistentes e assim respondendo melhor aos pontos de corte da teoria de modo duplo. Alcançando repostas afetivas positivas em qualquer intensidade do TR.

Como limitação, esta revisão sistemática teve como destaque 0 baixo número de artigos com a temática proposta. Por outro lado, estudos de grande validade juntamente com a teoria do modo duplo, e demais recomendações, corroboraram com a resposta eminente dessa presente revisão sistemática. Demonstrando que a intensidade deve ser prescrita levando em consideração não apenas as respostas fisiológicas como também as afetivas, para que haja uma maior aderência ao TR em idosos. Corroborando com as teorias comportamentais, onde respostas afetivas positivas contribuem para o engajamento inicial no TR (EKKEKAKIS, 2003; EKKEKAKIS et al., 2008) 
Treinamento resistido e idosos

\section{CONCLUSÃO}

Após a realização da presente revisão sistemática, pôde-se concluir que com a utilização de intensidades entre moderadas e altas no treinamento resistido foram obtidas percepções de afeto positivas entre os idosos. Essa relação positiva entre a intensidade e a resposta afetiva

entre os idosos, destaca que a intensidade deve ser prescrita considerando não apenas as respostas fisiológicas, como também as afetivas, já que a última delas está ligada aderência ao treinamento.

\section{REFERÊNCIAS}

ACSM. AMERICAN COLLEGE OF SPORTS MEDICINE. A quantidade e o tipo recomendados de exercícios para o desenvolvimento e a manutenção da aptidão cardiorrespiratória e muscular em adultos saudáveis. Revista Brasileira de Medicina do Esporte, Niterói, v. 4, n. 3, p. 96-106, jun. 1998.

ANTONINI, T. C.; LIBERALI, R.; CRUZ, I. B. M. Treinamento de força e morbidades geriátricas: uma revisão. Revista Brasileira de Prescrição e Fisiologia do Exercício, São Paulo, v. 4, n. 23, p. 514-524, set./out. 2010.

BLUMENTHAL, J. A. et. al. Cardiovascular and Behavioral Effects of Aerobic Exercise Training in Healthy Older Men and Women. Journal of Gerontology, Washington, V. 44, n. 5, p. M147-M157, set. 1989.

CASSILHAS, R. C. et al. Mood, anxiety, and serum IGF-1 in elderly men given 24 weeks of high resistance exercise. Perceptual and Motor skills, v. 110, n. 1, p. 265-276, fev. 2010.

EDWARDS, N. et al. Effect of exercise on negative affect in residents in special care units with moderate to severe dementia. Alzheimer Disease \& Associated Disorders, v. 22, n. 4, p. 362-368, dez. 2008.

EKKEKAKIS P, BACKHOUSE SH, GRAY C, ET AL. Walking is popular among adults but it is pleasant? A framework for clarifying the link between walking and affect as illustrated in two studies. Psychol Sport Exerc, v. 9, n. 2, p. 246-264, maio 2008.

EKKEKAKIS, P.; HALL, E.; PETRUZZELLO, S. J. Variation and homogeneity in affective responses to physical activity of varying intensities: an alternative perspective on dose-response based on evolutionary considerations. Journal of sports sciences, Ames, v. 23, n. 5, p. 477-500, mai. 2005.

EKKEKAKIS, P; LIND, E. Exercise does not feel the same when you are overweight: the impact of selfselected and imposed intensity on affect and exertion. International journal of obesity, Londres, v. 30, n. 4, p. 652-660, abr. 2006.

EKKEKAKIS P, PARFITT G, PETRUZZELLO SJ. The pleasure and displeasure people feel when they exercise at different intensities: decennial update and progress towards a tripartite rationale for exercise intensity prescription. Sports Med, v. 41, n. 8, p. 641-671, agos. 2011.

EKKEKAKIS, P. Pleasure and displeasure from the body: Perspectives from exercise. Cognition \& Emotion, Ames, v. 17, n. 2, p. 213-239, mar. 2003.

FECHINE, B. R. A.; TROMPIERI, N. O processo de envelhecimento: As principais alterações que acontecem com o idoso com o passar dos anos. Inter Science Place, Campos dos Goytacazes, v. 1, n. 7, p. 106-132, mar. 2012.

HARDY, C. J.; REJESKI, W. J. Not what, but how one feels: The measurement of affect during exercise. Journal of Sport and Exercise Psychology, Winston-Salem, v. 11, n. 3, p. 304-317, nov. 1989. 
JETTE, A. M. et al. A home-b

Treinamento resistido e idosos

: older adults. Journal of the

American Geriatrics Society, v. 44, n. 6, p. 644-64y, jun. 1 yyb.

LACHARITÉ, Lemieux; M., BRUNELLE, J. P. \& DIONNE, I. J. Adherence to exercise and affective responses: comparison between outdoor and indoor training. Menopause. The Journal of The North American Menopause Society, vol. 22, № 7, p. 731-740, mar. 2015.

MATSUDO, S. M.; MATSUDO, V. K. R.; BARROS NETO, T. L. Atividade física e envelhecimento: aspectos epidemiológicos. Revista Brasileira de Medicina do Esporte, Niterói, v. 7, n. 1, p. 2-13, dez. 2001.

MULLEN, S. P. et al. Measuring enjoyment of physical activity in older adults: invariance of the physical activity enjoyment scale (paces) across groups and time. International Journal of Behavioral Nutrition and Physical Activity, v. 8, n. 1, p. 103, set. 2011.

O'SHEA, S. D.; TAYLOR, N. F.; PARATZ, J. D. Qualitative outcomes of progressive resistance exercise for people with COPD. Chronic respiratory disease, v. 4, n. 3, p. 135-142, mar. 2015.

SCHNEIDER, R. H.; IRIGARAY, T. Q. O envelhecimento na atualidade: aspectos cronológicos, biológicos, psicológicos e sociais. Estudos de Psicologia, Campinas, v. 25, n. 4, p. 585-593, dez. 2008. TSUTSUMI, T. et al. Physical fitness and psychological benefits of strength training in community dwelling older adults. Applied human science, v. 16, n. 6, p. 257-266, nov. 1997.

TSUTSUMI, Toshihiko et al. Comparison of high and moderate intensity of strength training on mood and anxiety in older adults. Perceptual and motor skills, v. 87, n. 3, p. 1003-1011, dez. 1998.

ZACARON, K. A. M. et al. Nível de atividade física, dor e edema e suas relações com a disfunção muscular do joelho de idosos com osteoartrite. Revista Brasileira de Fisioterapia, São Carlos, v. 10, n. 3, p. 279-284, jul./set. 2006. 